\section{A-kikiog}

Archive for

Organic Chemistry
The Free Internet Journal

for Organic Chemistry
Paper

Arkivoc 2020, part vi, 0-0

\title{
An efficient synthesis of Nepetoidin B
}

\author{
Ming Yao ${ }^{a^{*}}$, Jingjing Zhang ${ }^{a, b}$, Sen Yang ${ }^{a}$, and Hangxing Xiong a, ${ }^{*}$ \\ a Jingchu University of Technology, Jingmen and 448000, P. R. China \\ ${ }^{b}$ Wuhan Institute of Technology, Wuhan and 430205, P. R. China \\ Email:yaomingcep@jcut.edu.cn
}

Received 12-05-2019

Accepted 01-19-2020

Published on line $\quad 03-25-2020$

\section{Abstract}

The synthesis of nepetoidin B from readily and commercially available 3,4-dimethoxybenzaldehyde in an overall yield of $52 \%$ is achieved. The present three-step protocol involves Ramirez-Corey-Fuchs reaction, ruthenium-catalyzed anti-Markovnikov addition of a carboxylic acid to a terminal alkyne and demethylation using iodotrimethylsilane ( $\mathrm{Me}_{3} \mathrm{Sil}$ ) and quinoline. In this work, the two isomers of $(Z, E)$ - nepetoidin $\mathrm{B} /(E, E)$ nepetoidin $B$ and the single isomer of $(E, E)$ - nepetoidin $B$ can be obtained through the demethylation of $(Z, E)$ 5 and $(E, E)-5$, respectively.

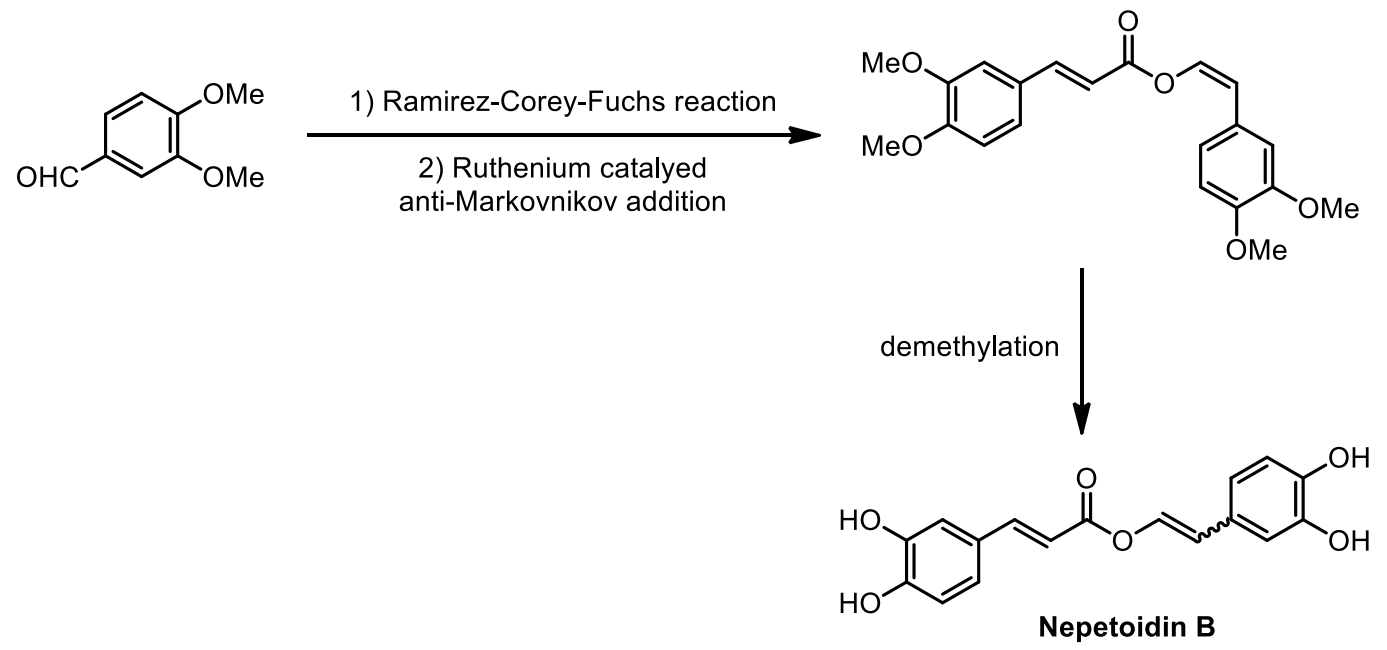

Keywords: Nepetoidin B, 3,4-dimethoxybenzaldehyde, terminal alkyne, anti-Markovnikov addition, demethylation, synthesis 


\section{Introduction}

Nepetoidin B (1) is a phenolic compound with $(Z, E)-\mathbf{1}$ and $(E, E)-\mathbf{1}$ isomers (Figure 1). It could be isolated from Perilla frutescens, ${ }^{1}$ Plectranthus forsteri, ${ }^{2-3}$ Salvia miltiorrhiza Bunge ${ }^{4}$ and other plants. ${ }^{5-14}$ The amounts of $(Z$, $E)-1$ and $(E, E)$-1 vary from 9:1 to 1:1 in different plants. Nepetoidin B possesses remarkable biological activities, such as anti-oxidant, anti-viral and anti-fungal activities, and it could also be used to inhibit xanthine oxidase and the production of nitric oxide. ${ }^{4,8,11}$
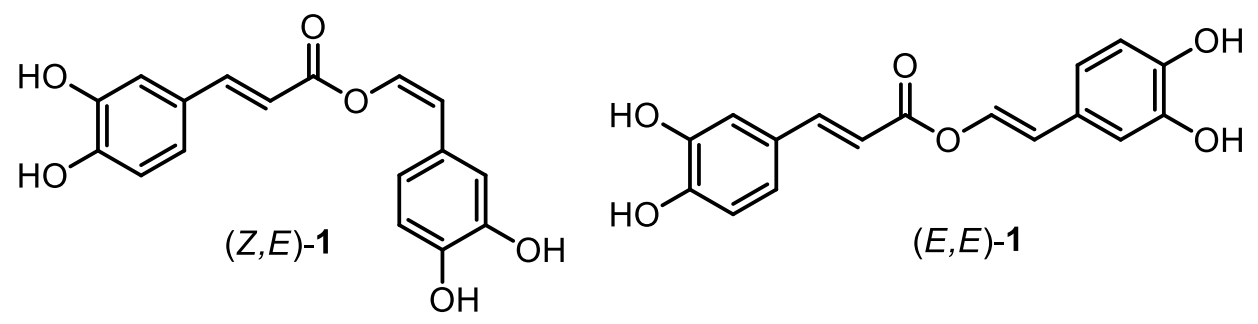

Figure 1. The two isomers of nepetoidin B.

The level of nepetoidin B in plants is rather low. $100 \mathrm{~kg}$ of Salvia miltiorrhiza Bunge (Danshen) could only provide $37 \mathrm{mg}$ nepetoidin B. ${ }^{4}$ It would hardly be possible to provide large quantities of nepetoidin B by extraction to conduct further biological research. Therefore, efficient synthetic approaches for large-scale preparation of nepetoidin B are highly desired. The first synthesis of nepetoidin B was developed by Timokhin et al. through Baeyer-Villiger oxidation of 1,5-bis(3,4-dimethoxyphenyl)-1,4-pentadien-3-one with oxone and demethylation with boron tribromide. The overall yield of this approach was $17 \%{ }^{15}$ The first step of this approach required a tedious workup and purification to afford tetramethylated nepetoidin B in $40 \%$ yield and to recover the starting material. ${ }^{15}$

Due to our interest in the synthesis of biological active phenolic compounds, ${ }^{16-20}$ we developed an efficient route for the synthesis of nepetoidin B in three steps from the commercially available and inexpensive 3,4dimethoxybenzaldehyde.

\section{Results and Discussion}

As illustrated in Figure 2, we undertook a retrosynthetic analysis of nepetoidin B. First, demethylation of the tetramethylated nepetoidin B could afford nepetoidin B. Further disconnection at the 010-C11 bond of tetramethylated nepetoidin B provided two fragments $\mathbf{3}$ and $\mathbf{4}$, which could be forged together by a Rucatalyzed anti-Markovnikov addition of the carboxylic acid to the terminal alkyne. The carboxylic acid $\mathbf{4}$ and alkyne 3 could be prepared from 3,4-dimethoxybenzaldehyde.

As shown in figure 3, the synthesis commenced by preparing the 3,4-dimethoxyphenylacetylene 3 . Ramirez-Corey-Fuchs reaction of 3,4-dimethoxybenzaldehyde afforded the corresponding terminal alkyne $\mathbf{3}$ in $95 \%$ yield. ${ }^{21-22}$ The 3,4-dimethoxycinnamic acid is a very cheap raw material, although it can by prepared via a Knoevenagel-Doebner condensation in high yield. ${ }^{23}$ Subsequently, we employed the Ru-catalyzed antiMarkovnikov addition reaction developed by Dixneuf et al. ${ }^{24}$ to prepare the tetramethylated nepetoidin B 5 from 3,4-dimethoxyphenylacetylene 3 and 3,4-dimethoxycinnamic acid $\mathbf{4}$. In the presence of [Ru]cat., the addition occurred smoothly to afford compound $\mathbf{5}$ in $90 \%$ yield at $45{ }^{\circ} \mathrm{C}$, with toluene as solvent. Delightfully, we obtained the single isomer as $(E, Z)-5$ and did not observe the isomer of $(E, Z)-5$ (as determined by ${ }^{1} \mathrm{H}-\mathrm{NMR}$ 
and ${ }^{13} \mathrm{C}-\mathrm{NMR}$ analysis). Replacement of the toluene with THF or hexane resulted in a poor yield of the desired product. Efforts to increase the reaction temperature to $60^{\circ} \mathrm{C}$ or decrease the temperature to $20{ }^{\circ} \mathrm{C}$ only led to low yields.

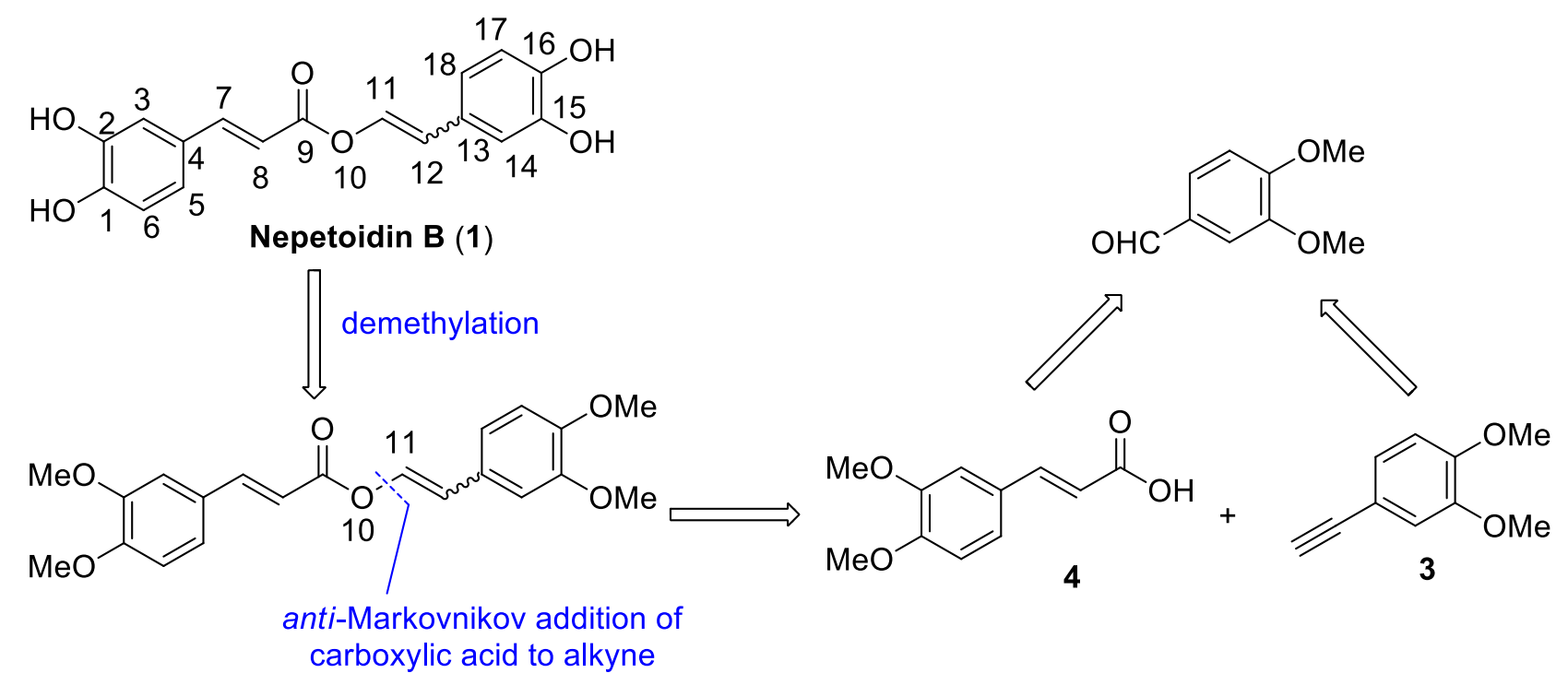

Figure 2. Retrosynthetic analysis of nepetoidin B.

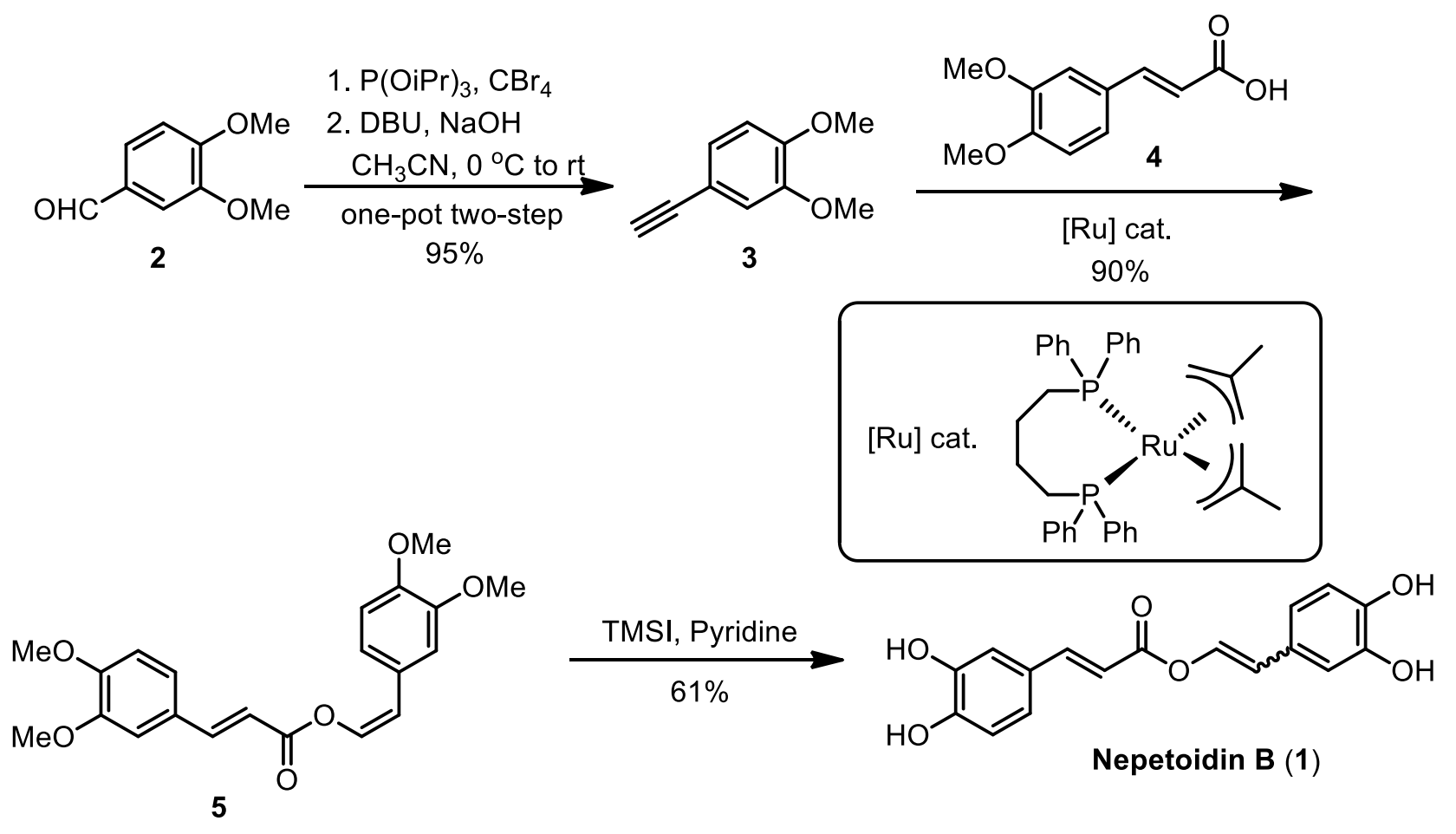

Figure 3. Synthesis of nepetoidin B

The final step in the synthesis required the demethylation of compound 5. Though Timokhin et al. ${ }^{15}$ used boron tribromide to conduct the demethylation of compound 5 , the isolated yield of this method was $43 \%$ and the obtained compound 1 was a mixture of the two isomers in a ratio of 94:6 $(E, E)-\mathbf{1} /(Z, E)-\mathbf{1}$. Therefore, we further examined various demethylation methods as shown in Table $1 .^{25-26}$ Treatment of compound $(Z, E)-5$ 
with $\mathrm{Me}_{3} \mathrm{Sil}$ and quinoline at $175{ }^{\circ} \mathrm{C}$ for $4 \mathrm{~h}$ afforded the desired product in $61 \%$ yield as a mixture of the two isomers in a ratio of 2.8:1 (Z,E)-1/(E, E)-1 (as estimated by ${ }^{1} \mathrm{H}-\mathrm{NMR}$ analysis)(entry 7 ). However, treatment of the compound $(E, E)-\mathbf{5}$ under the same conditions could provide a single isomer of $(E, E)-\mathbf{1}$ in $59 \%$ yield (entry 7). The clear reason for the formation of $(E, E)-\mathbf{1}$ is unclear. The reaction temperature $\left(175^{\circ} \mathrm{C}\right)$ is perhaps the reason for the partial isomerisation of the double bond. In addition, we also probed other demethylation methods, but low yields of compound 1 (entry 6), complex products (entry 3-5) or ester bond (C9-010) cleavage products (entry 1-2) were obtained.

Table 1. Demethylation of compound $5^{a}$

\begin{tabular}{clc}
\hline Entry & \multicolumn{1}{c}{ Reagents and Conditions } & Yield/\% \\
\hline 1 & $46 \% \mathrm{HBr}, \mathrm{TBAB}$ or $\mathrm{nBu} 4 \mathrm{PBr}$, reflux & $-{ }^{b}$ \\
2 & $\mathrm{All}_{3}, \mathrm{CH}_{3} \mathrm{CN}, \mathrm{rt}$ or $75^{\circ} \mathrm{C}$ & $-{ }^{b}$ \\
3 & $\mathrm{Me}_{3} \mathrm{Sil}, \mathrm{CHCl}, \mathrm{rt}$ & $-c$ \\
4 & $\mathrm{Nal}_{3} \mathrm{Me}_{3} \mathrm{SiCl}, \mathrm{CH}_{3} \mathrm{CN}$ & $-c$ \\
5 & $\mathrm{BF}_{3} \bullet \mathrm{Et}_{2} \mathrm{O}, \mathrm{SHCH}_{2} \mathrm{CH}_{2} \mathrm{SH}, \mathrm{CH}_{2} \mathrm{Cl}_{2}, \mathrm{rt}$ & $-{ }^{c}$ \\
6 & $\mathrm{All}_{3}, \mathrm{DIC}, \mathrm{CH}_{3} \mathrm{CN}, \mathrm{rt}$ or $75^{\circ} \mathrm{C}$ & $<5$ \\
7 & MeSil, quinoline, $4 \mathrm{~h}, 175^{\circ} \mathrm{C}$ & $61^{d}(59)^{e}$ \\
\hline
\end{tabular}

a Unless noted otherwise, all the reaction were conducted with compound $(Z, E)-5(0.4 \mathrm{mmol})$ in the presence of the specified demethylation methods; ${ }^{b}$ The major products were the ester bond (C9-010) cleavage products; ${ }^{c}$ complex products; $d$ The obtained product was as a mixture of the two isomers in a ratio of 2.8:1 $(Z, E)-1 /(E$, $E)-1 ;{ }^{e}$ The $(E, E)-5$ was used as the starting material. Value in parentheses was the isolated yield of $(E, E)-\mathbf{1}$. $\mathrm{TBAB}=$ Tetrabutylammonium bromide; $\mathrm{DIC}=1,3-$ diisopropylcarbodiimide.

\section{Conclusions}

In conclusion, we have developed a concise approach to the synthesis of nepetoidin B in three steps and $52 \%$ overall yield from commercially available 3,4-dimethoxybenzaldehyde. In the presence of $\mathrm{Me}_{3} \mathrm{Sil}$ and quinoline at $175{ }^{\circ} \mathrm{C}$, the $(Z, E)-\mathbf{5}$ could afford two isomers of $(Z, E)-\mathbf{1} /(E, E)-\mathbf{1}$ (the ratio of $(Z, E)-\mathbf{1} /(E, E)-\mathbf{1}$ was $\left.2.8: 1\right)$ in $61 \%$ yield and the $(E, E)-5$ could afford the single isomer of $(E, E)-1$ in $59 \%$ yield. This protocol offered a straightforward and sustainable access to nepetoidin B from readily and commercially available starting materials with high efficiency.

\section{Experimental Section}

General. Commercial reagents and solvents were used as received without further purification. $\mathrm{CH}_{3} \mathrm{CN}$ was of $\mathrm{HPLC}$ grade with less than $500 \mathrm{ppm}$ of $\mathrm{H}_{2} \mathrm{O}$. All 3 was prepared in situ by mixing Al powder and $\mathrm{I}_{2 .}{ }^{27}$ [Ru]cat. was 
prepared by displacement of cyclooctadiene from bis(2-methylpropenyl)(cycloocta-1,5-diene)ruthenium by 1 equiv of the 1,4-bis(diphenylphosphino)butane according to Genet's method. ${ }^{28}$ The $(E, E)-5$ was synthesized according to the procedure developed by Timokhin et al. ${ }^{15}$ TLC analyses were performed on precoated GF254 silica gel plates and were visualized under UV254 nm light or by 12 staining. NMR spectra were detected using a Bruker Avance 400 FTNMR spectrometer. Column chromatography was carried out using 200-300 mesh silica gel.

One-pot two-step procedure for the preparation of 3,4-Dimethoxyphenylacetylene (3). To a stirred solution of 3,4-dimethoxybenzaldehyde 2 ( $831 \mathrm{mg}, 5.0 \mathrm{mmol})$ and tetrabromomethane $(2.49 \mathrm{~g}, 7.5 \mathrm{mmol})$ in $\mathrm{CH}_{3} \mathrm{CN}_{(10}$ $\mathrm{mL})$, triisopropyl phosphate $(2.5 \mathrm{~mL}, 10.0 \mathrm{mmol})$ was added drop wise over a period of $20 \mathrm{~min}$ at $0{ }^{\circ} \mathrm{C}$. After 15 $\mathrm{min}, \mathrm{DBU}(3.0 \mathrm{~mL}, 20.0 \mathrm{mmol})$ was added to the reaction mixture drop wise over 20 min while maintaining the temperature of the reaction at $0{ }^{\circ} \mathrm{C}$. The reaction mixture was then warmed to $25^{\circ} \mathrm{C}$ over 30 min. Powdered $\mathrm{NaOH}(50 \mathrm{mmol}$ ) was added to the reaction mixture in portion. After completion of the reaction (usually $5 \mathrm{~h}$ ), $50 \mathrm{~mL}$ water was added to the reaction mixture. The reaction mixture was extracted with EtOAc $(50 \mathrm{~mL})$ for three times and the organic layers were washed with saturated $\mathrm{NaCl}$ aq. $(60 \mathrm{~mL})$. The organic layers were dried with $\mathrm{Na}_{2} \mathrm{SO}_{4}$ and evaporated under reduced pressure to provide a crude residue, which was purified via using petroleum/EtOAc (20:1) as eluent to afford the terminal alkyne $\mathbf{3}$ as a white solid (770 mg, 95\% yield). ${ }^{1} \mathrm{H}-\mathrm{NMR}\left(\mathrm{CDCl}_{3}, 400 \mathrm{~Hz}\right): \delta 7.10(\mathrm{dd}, J 8.0,2.0 \mathrm{~Hz}, 1 \mathrm{H}), 6.99(\mathrm{~d}, J 2.0 \mathrm{~Hz}, 1 \mathrm{H}), 6.80(\mathrm{~d}, J 8.0 \mathrm{~Hz}, 1 \mathrm{H}), 3.89(\mathrm{~s}, 3 \mathrm{H})$, $3.88(\mathrm{~s}, 3 \mathrm{H}), 3.00(\mathrm{~s}, 1 \mathrm{H}){ }^{29}$

(Z,E)-Tetramethylated nepetoidin B (5). 3,4-Dimethoxycinnamic acid 4 (416 mg, 2.0 mmol), 3,4Dimethoxyphenylacetylene 3 (324 mg, $2.0 \mathrm{mmol}$ ) and [Ru]cat. (12.8 mg, $0.02 \mathrm{mmol})$ in $10 \mathrm{~mL}$ of toluene were stirred at $45{ }^{\circ} \mathrm{C}$ for $12 \mathrm{~h}$ under an inert atmosphere of nitrogen. The reaction mixture was quenched with $\mathrm{NaHCO}_{3}$ aq. $(10 \mathrm{~mL})$ and extracted with EtOAc $(20 \mathrm{~mL})$ for three times. The organic layers were washed with $\mathrm{NaCl}$ aq. $\left(30 \mathrm{~mL}\right.$ ). The organic layers were dried with $\mathrm{Na}_{2} \mathrm{SO}_{4}$ and evaporated under reduced pressure to provide a crude residue, which was purified via using petroleum/EtOAc (3:1) as eluent to afford the tetramethylated nepetoidin B 5 as a yellow solid $\left(667 \mathrm{mg}, 90 \%\right.$ yield). $(Z, E)-5: \mathrm{mp} 109-110{ }^{\circ} \mathrm{C},{ }^{1} \mathrm{H}-$ NMR(acetone $\left.d_{6}, 400 \mathrm{~Hz}\right): \delta 7.82(\mathrm{~d}, J 16 \mathrm{~Hz}, 1 \mathrm{H}), 7.38(\mathrm{~d}, J 7.2 \mathrm{~Hz}, 1 \mathrm{H}), 7.28(\mathrm{~d}, J 2.0 \mathrm{~Hz}, 1 \mathrm{H}), 7.21(\mathrm{dd}, J 8.4,2.0$ $\mathrm{Hz}, 1 \mathrm{H}), 7.15(\mathrm{dd}, J$ 8.4, $2.0 \mathrm{~Hz}, 1 \mathrm{H}), 7.08(\mathrm{~d}, J 2.0 \mathrm{~Hz}, 1 \mathrm{H}), 6.91(\mathrm{~d}, J 2.8 \mathrm{~Hz}, 1 \mathrm{H}), 6.89(\mathrm{~d}, J 2.8 \mathrm{~Hz}, 1 \mathrm{H}), 6.42(\mathrm{~d}, J$ $16 \mathrm{~Hz}, 1 \mathrm{H}), 5.72(\mathrm{~d}, J 7.2 \mathrm{~Hz}, 1 \mathrm{H}), 3.94(\mathrm{~s}, 3 \mathrm{H}), 3.94(\mathrm{~s}, 3 \mathrm{H}), 3.93(\mathrm{~s}, 3 \mathrm{H}), 3.92(\mathrm{~s}, 3 \mathrm{H}){ }^{12}{ }^{13} \mathrm{C}-\mathrm{NMR}($ acetone $\left.d_{6}, 100 \mathrm{~Hz}\right): \delta 164.2,153.1,150.6,145.0,149.7,147.6,133.5,128.2,127.9,124.1,123.1,114.8,113.7,112.5$, $112.5,112.3,111.3,56.1,56.1,56.0,56.0$.

Nepetoidin B $(Z, E)-1 /(E, E)-1$. To a stirred solution of quinoline $(5.0 \mathrm{~mL})$ in a $10 \mathrm{~mL}$ round bottom flask, Me 3 Sil $(1.0 \mathrm{~mL})$ was added drop wise at $0{ }^{\circ} \mathrm{C}$ under an inert atmosphere of nitrogen. After 15 min, tetramethylated nepetoidin $B(Z, E)-5$ (148 mg, $0.4 \mathrm{mmol})$ was added quickly. The reaction mixture was then warmed to $175{ }^{\circ} \mathrm{C}$ and stirred for $4 \mathrm{~h}$ at this temperature. After the reaction mixture was cooled to $\mathrm{rt}$, it was quenched with $\mathrm{HCl}$ $(30 \mathrm{~mL}, 1.0 \mathrm{~mol} / \mathrm{L})$ and stirred for $1 \mathrm{~h}$. The reaction mixture was extracted with EtOAc $(30 \mathrm{~mL})$ for three times. The organic layers were washed with $\mathrm{NaCl}$ aq. $(30 \mathrm{~mL})$. The organic layers were dried with $\mathrm{MgSO}_{4}$ and evaporated under reduced pressure to provide a crude residue, which was purified via using $\mathrm{CH}_{2} \mathrm{Cl}_{2} / \mathrm{MeOH}$ $(15: 1)$ as eluent to afford the nepetoidin $B(Z, E)-\mathbf{1} /(E, E)-\mathbf{1}$ as a yellow solid $(77 \mathrm{mg}, 61 \%$ yield). The ratio of isomers $(Z, E)-\mathbf{1} /(E, E)-\mathbf{1}$ is 2.8:1, as determined by ${ }^{1} \mathrm{H}-\mathrm{NMR}$ spectroscopic analysis (signals at $7.75(\mathrm{~d}, J 16 \mathrm{~Hz}$, $1 \mathrm{H}, \mathrm{H}-7)$ and $7.82(\mathrm{~d}, J 12.8 \mathrm{~Hz}, 1 \mathrm{H}, \mathrm{H}-11)$ for the $(\mathrm{Z}, \mathrm{E})-1$ and $(\mathrm{E}, \mathrm{E})-1$ isomers, respectively).

$(Z, E)-1:{ }^{1} \mathrm{H}-\mathrm{NMR}\left(\right.$ acetone $\left.d_{6}, 400 \mathrm{~Hz}\right): \delta 7.75(\mathrm{~d}, J 16 \mathrm{~Hz}, 1 \mathrm{H}), 7.38(\mathrm{~d}, J 2.0 \mathrm{~Hz}, 1 \mathrm{H}), 7.27(\mathrm{~d}, J 7.2 \mathrm{~Hz}, 1 \mathrm{H}), 7.26(\mathrm{~d}$, J $2.0 \mathrm{~Hz}, 1 \mathrm{H}), 7.14(\mathrm{dd}, J 8.2,2.0 \mathrm{~Hz}, 1 \mathrm{H}), 7.02(\mathrm{dd}, J 8.0,2.0 \mathrm{~Hz}, 1 \mathrm{H}), 6.91(\mathrm{~d}, J 8.4 \mathrm{~Hz}, 1 \mathrm{H}), 6.83(\mathrm{~d}, J 8.4 \mathrm{~Hz}, 1 \mathrm{H})$, $6.51(\mathrm{~d}, J 16 \mathrm{~Hz}, 1 \mathrm{H}), 5.68(\mathrm{~d}, J 7.2 \mathrm{~Hz}, 1 \mathrm{H}) ;{ }^{13} \mathrm{C}-\mathrm{NMR}$ (acetone $\left.d_{6}, 100 \mathrm{~Hz}\right): \delta 164.4,149.2,148.0,146.3,145.5$, 
$145.5,132.9,127.5,127.4,123.2,122.6,117.0,116.4,116.0,115.4,114.0,112.5 .(E, E)-1:{ }^{1} \mathrm{H}-\mathrm{NMR}\left(\right.$ acetone $d_{6}$, $400 \mathrm{~Hz}): \delta 7.82(\mathrm{~d}, J 12.8 \mathrm{~Hz}, 1 \mathrm{H}), 7.70(\mathrm{~d}, J 16 \mathrm{~Hz}, 1 \mathrm{H}), 7.23(\mathrm{~d}, J 2.0 \mathrm{~Hz}, 1 \mathrm{H}), 7.12(\mathrm{dd}, J 8.0,2.0 \mathrm{~Hz}, 1 \mathrm{H}), 6.94(\mathrm{~m}$, $1 \mathrm{H}), 6.90(\mathrm{~d}, J 8.4 \mathrm{~Hz}, 1 \mathrm{H}), 6.79(\mathrm{~m}, 2 \mathrm{H}), 6.38(\mathrm{~d}, J 12.8 \mathrm{~Hz}, 1 \mathrm{H}), 6.37(\mathrm{~d}, J 16 \mathrm{~Hz}, 1 \mathrm{H})$; ${ }^{13} \mathrm{C}-\mathrm{NMR}($ acetone $d_{6,100 \mathrm{~Hz}):} \delta 164.8,149.2,147.7,146.3,146.1,145.7,135.4,127.4,127.3,123.1,119.3,116.4,116.4,115.9$, $115.4,113.9,113.6 .^{5,15}$

$(Z, E)-1:{ }^{1} \mathrm{H}-\mathrm{NMR}\left(\mathrm{CD}_{3} \mathrm{OD}, 400 \mathrm{~Hz}\right): \delta 7.72(\mathrm{~d}, J 16 \mathrm{~Hz}, 1 \mathrm{H}), 7.30(\mathrm{~d}, J 2.0 \mathrm{~Hz}, 1 \mathrm{H}), 7.23(\mathrm{~d}, J 7.2 \mathrm{~Hz}, 1 \mathrm{H}), 7.13(\mathrm{~d}, J$ $2.0 \mathrm{~Hz}, 1 \mathrm{H}), 7.03(\mathrm{dd}, J$ 8.0, $2.0 \mathrm{~Hz}, 1 \mathrm{H}), 6.91(\mathrm{dd}, J 8.4,2.0 \mathrm{~Hz}, 1 \mathrm{H}), 6.82(\mathrm{~d}, J 8.0 \mathrm{~Hz}, 1 \mathrm{H}), 6.76(\mathrm{~d}, J 8.4 \mathrm{~Hz}, 1 \mathrm{H})$, $6.45(\mathrm{~d}, J 16 \mathrm{~Hz}, 1 \mathrm{H}), 5.62$ (d, J $7.2 \mathrm{~Hz}, 1 \mathrm{H}) ;{ }^{13} \mathrm{C}-\mathrm{NMR}\left(\mathrm{CD}_{3} \mathrm{OD}, 100 \mathrm{~Hz}\right): \delta 165.7,149.8,148.8,148.8,146.6,145.8$, $132.9,127.8,127.5,123.4,122.8,117.2,116.5,116.1,115.5,113.6,113.1 .(E, E)-1:{ }^{1} \mathrm{H}-\mathrm{NMR}\left(\mathrm{CD}_{3} \mathrm{OD}, 400 \mathrm{~Hz}\right): \delta$ $=7.79(\mathrm{~d}, J=12.8 \mathrm{~Hz}, 1 \mathrm{H}), 7.66(\mathrm{~d}, J=15.6 \mathrm{~Hz}, 1 \mathrm{H}), 7.09(\mathrm{~d}, J=2.4 \mathrm{~Hz}, 1 \mathrm{H}), 6.99(\mathrm{dd}, J 8.2,2.0 \mathrm{~Hz}, 1 \mathrm{H}), 6.84(\mathrm{~d}, J$ $1.6 \mathrm{~Hz}, 1 \mathrm{H}), 6.80(\mathrm{~d}, J 8.0 \mathrm{~Hz}, 1 \mathrm{H}), 6.73-6.70(\mathrm{~m}, 2 \mathrm{H}), 6.35(\mathrm{~d}, J 12.8 \mathrm{~Hz}, 1 \mathrm{H}), 6.32(\mathrm{~d}, J 15.6 \mathrm{~Hz}, 1 \mathrm{H}) ;{ }^{13} \mathrm{C}-$ $\mathrm{NMR}\left(\mathrm{CD}_{3} \mathrm{OD}, 100 \mathrm{~Hz}\right): \delta 166.3,149.7,148.4,146.6,146.3,146.0,145.8,135.5,119.7,116.6,116.5,115.3$, 113.6. ${ }^{8}$

\section{Nepetoidin B $(E, E)-1$}

To a stirred solution of quinoline $(5.0 \mathrm{~mL})$ in a $10 \mathrm{~mL}$ round bottom flask, Me ${ }_{3} \mathrm{Sil}(1.0 \mathrm{~mL})$ was added drop wise at $0{ }^{\circ} \mathrm{C}$ under an inert atmosphere of nitrogen. After $15 \mathrm{~min}$, tetramethylated nepetoidin $\mathrm{B}(E, E)-5(148 \mathrm{mg}$, $0.4 \mathrm{mmol}$ ) was added quickly. The reaction mixture was then warmed to $175^{\circ} \mathrm{C}$ and stirred for $4 \mathrm{~h}$ at this temperature. After the reaction mixture was cooled to $\mathrm{rt}$, it was quenched with $\mathrm{HCl}(30 \mathrm{~mL}, 1.0 \mathrm{~mol} / \mathrm{L})$ and stirred for $1 \mathrm{~h}$. The reaction mixture was extracted with EtOAc $(30 \mathrm{~mL})$ for three times. The organic layers were washed with $\mathrm{NaCl}$ aq. (30 mL). The organic layers were dried with $\mathrm{MgSO}_{4}$ and evaporated under reduced pressure to provide a crude residue, which was purified via using $\mathrm{CH}_{2} \mathrm{Cl}_{2} / \mathrm{MeOH}(15: 1)$ as eluent to afford the nepetoidin $B(E, E)-1$ as a yellow solid $\left(74 \mathrm{mg}, 59 \%\right.$ yield). ${ }^{1} \mathrm{H}-\mathrm{NMR}$ (acetone $\left.d_{6}, 400 \mathrm{~Hz}\right): \delta 8.61(\mathrm{~s}, 1 \mathrm{H}), 8.29(\mathrm{~s}$, $1 \mathrm{H}), 8.01(\mathrm{~s}, 1 \mathrm{H}), 7.95(\mathrm{~s}, 1 \mathrm{H}), 7.83(\mathrm{~d}, J 12.8 \mathrm{~Hz}, 1 \mathrm{H}), 7.70(\mathrm{~d}, J 16 \mathrm{~Hz}, 1 \mathrm{H}), 7.23(\mathrm{~d}, J 2.0 \mathrm{~Hz}, 1 \mathrm{H}), 7.12(\mathrm{dd}, J 8.2$, $2.0 \mathrm{~Hz}, 1 \mathrm{H}), 6.91(\mathrm{~m}, 2 \mathrm{H}), 6.79(\mathrm{~m}, 2 \mathrm{H}), 6.38(\mathrm{~d}, J 12 \mathrm{~Hz}, 1 \mathrm{H}), 6.37(\mathrm{~d}, J 16.4 \mathrm{~Hz}, 1 \mathrm{H})$; ${ }^{13} \mathrm{C}-\mathrm{NMR}($ acetone $\left.d_{6}, 100 \mathrm{~Hz}\right): \delta 164.8,149.3,147.7,146.4,146.2,145.8,135.4,127.4,127.3,123.1,119.3,116.4,116.4,115.9$, 115.4, 113.9, 113.6. ${ }^{5,15}{ }^{1} \mathrm{H}-\mathrm{NMR}\left(\mathrm{DMSO} d_{6}, 400 \mathrm{~Hz}\right): \delta 9.17$ (br. s, 4H), 7.71 (d, J $\left.12.8 \mathrm{~Hz}, 1 \mathrm{H}\right), 7.64$ (d, J $15.8 \mathrm{~Hz}$, $1 \mathrm{H}), 7.12(\mathrm{~d}, J 2.0 \mathrm{~Hz}, 1 \mathrm{H}), 7.07(\mathrm{dd}, J 8.2,2.0 \mathrm{~Hz}, 1 \mathrm{H}), 6.82(\mathrm{~s}, 1 \mathrm{H}), 6.79(\mathrm{~d}, J 8.1 \mathrm{~Hz}, 1 \mathrm{H}), 6.69(\mathrm{~m}, 2 \mathrm{H}), 6.37(\mathrm{~d}, J$ $12.8 \mathrm{~Hz}, 1 \mathrm{H}), 6.36(\mathrm{~d}, J 16 \mathrm{~Hz}, 1 \mathrm{H}) ;{ }^{13} \mathrm{C}-\mathrm{NMR}\left(\mathrm{DMSO} d_{6}, 100 \mathrm{~Hz}\right): \delta 164.1,149.0,147.2,145.69,145.5,145.2$, $134.2,125.4,125.1,12.0,118.0,159.9,115.8,115.4,115.1,113.1,112.3$.

\section{Acknowledgements}

We thank Dr. Dayong Sang for helpful discussions. Financial support was provided by the Hubei Provincial Department of Education (T201719).

\section{Supplementary Material}

Characterization data, copies of ${ }^{1} \mathrm{H}$ and ${ }^{13} \mathrm{C}$ NMR spectra associated with article can be found in the online version. 


\section{References}

1. Nakanishi, T.; Nishi, M.; Inada, A.; Obata, H.; Tanabe, N.; Abe, S.; Wakashiro, M. Chem. Pharm. Bull. 1990, $38,1772$.

https://doi.org/10.1248/cpb.38.1772

2. Kubinova, R.; Svajdlenka, E.; Schneiderova, K.; Hanakova, Z.; Dall'Acqua, S.; Farsa, O. Biochem. Syst. Ecol. 2013, 49, 39.

https://doi.org/10.1016/j.bse.2013.03.029

3. Arihara, S.; Ruedi, P.; Eugster, C. H. Helv. Chim. Acta. 1975, 58, 447.

https://doi.org/10.1002/hlca.19750580214

4. Wu, X.; Gao, H.; Sun, W.; Yu, J.; Hu, H.; Xu, Q.; Chen, X. Phytother. Res. 2017, 31, 1072.

https://doi.org/10.1002/ptr.5828

5. Zhou, W.; Xie, H.; Xu, X.;Liang, Y.; Wei, X. J. Funct. Foods 2014, 6, 492.

https://doi.org/10.1016/i.jff.2013.11.015

6. Wang, G.; Li, T.; Deng, F.; Li, Y. Bioorg. Med. Chem. Lett. 2013, 23. 1379.

https://doi.org/10.1016/i.bmcl.2012.12.077

7. Murata, T.; Miyase, T.; Yoshizaki, F. J. Nat. Med. 2011, 65, 385.

https://doi.org/10.1007/s11418-010-0501-y

8. Tsai, S. F.; Lee, S. S. Phytochemistry 2014, 101,121.

https://doi.org/10.1016/j.phytochem.2014.01.016

9. Banthorpe, D. V.; Bilyard, H. J.; Brown, G. D. Phytochemistry 1989, 28, 2109.

https://doi.org/10.1016/S0031-9422(00)97929-9

10. Huang, Z.; Huang, B.; Xiao, C.; Dong, X.; Jiang, B. Nat. Prod. Res. 2015, 29, 628.

https://doi.org/10.1080/14786419.2014.980248

11. Grayer, R. J.; Eckert, M. R.; Veitch, N. C.; Kite, G. C.; Marin, P. D.; Kokubun, T.; Simmonds, M. S. J.; Paton, A. J. Phytochemistry 2003, 64, 519. https://doi.org/10.1016/S0031-9422(03)00192-4

12. Banthorpe, D. V.; Bilyard, H. J.; Watson, D. G. Phytochemistry 1985, 24, 2677. https://doi.org/10.1016/S0031-9422(00)80692-5

13. Fraga, B. M.; Gonzalez-Coloma, A.; Alegre-Gomez, S.; Lopez-Rodriguez, M.; Amador, L. J.; Diaz, C. E. Phytochemistry 2017, 133, 59.

https://doi.org/10.1016/i.phytochem.2016.10.008

14. Zhong, J.; Feng, Y.; Li, H.; Xia, X.; Li, R. Nat. Prod. Res. 2016, 30, 2278.

https://doi.org/10.1080/14786419.2016.1164698

15. Timokhin, V.; Regner, M.; Tsuji, Y.; Grabber, J.; Raoph, J. Synlett 2018, 29, 1229. https://doi.org/10.1055/s-0036-1591556

16. Tian, J.; Yue, H.; Yang, P.; Sang, D. ChemistrySelect 2019, 4, 38. https://doi.org/10.1002/slct.201803469

17. Sang, D.; Tian, J.; Tu, X.; He, Z.; Yao, M. Synthesis 2019, 51, 704. https://doi.org/10.1055/s-0037-1610996

18. Sang, D.; Tu, X.; Tian, J.; He, Z.; Yao, M. ChemistrySelect 2018, 3, 10103. https://doi.org/10.1002/slct.201802565

19. Sang, D.; Yao, M.; Tian, J.; Chen, X.; Zhan, H.; You, L. Synlett 2017, 28, 138.

https://doi.org/10.1055/s-0035-1588889 
20. Zhang, J.; Yao, M.; Li, L.; Sang, D.; Xiong, H.; Liu, S. Chin. J. Org. Chem. doi: 10.6023/cjoc201908014.

21. Thummala, Y.; Karunakar, G. V.; Doddi, V. R. Adv. Synth. Catal. 2019, 361, 611. https://doi.org/10.1002/adsc.201801334

22. Morri, A. K.; Thummala, Y.; Doddi, V. R. Org. Lett. 2015, 17, 4640. https://doi.org/10.1021/acs.orglett.5b02398

23. Mitra, A. K.; De, A.; Karchaudhuri, N. Synth. Commun. 1999, 29, 573. https://doi.org/10.1080/00397919908085805

24. Doucet, H.; Martin-Vaca, B.; Bruneau, C.; Dixneuf, P. H. J. Org. Chem. 1995, 60, 7247. https://doi.org/10.1021/jo00127a033

25. Yuan, H.; Shan, L.; Sun, Q.; Han, W. Acta Chim. Sinica 2011, 69, 945.

26. Junichi, M.; Brossi, A. Tetrahedron Lett. 1978, 34, 3085.

27. Sang, D.; Wang, J.; Zheng, Y.; He, J.; Yuan, C.; An, Q.; Tian, J. Synthesis 2017, 49, 2721. https://doi.org/10.1055/s-0036-1588755

28. Genet, J. P.; Mallart, S.; Pinel, C.; Juge, S.; Laffitte, J. A. Tetrahedron: Asymmetry 1991, 2, 43. https://doi.org/10.1016/S0957-4166(00)82155-2

29. Hosseini, A.; Pilevar, A.; Hogan, E.; Mogwitz, B.; Schulze, A. S.; Schreiner, P. R. Org. Biomol. Chem. 2017, 15,6800 .

https://doi.org/10.1039/C7OB01334E 